\title{
Philosophiques
}

\section{Ronald Dworkin, "Assisted Suicide : What the Court Really Said ", The New York Review of Books, 25 septembre 1997, p. 40-44.}

\section{Pierre-Yves Bonin}

Volume 25, numéro 2, automne 1998

Les modèles d'évolution en économie et en sciences sociales

URI : https://id.erudit.org/iderudit/027499ar

DOI : https://doi.org/10.7202/027499ar

Aller au sommaire du numéro

Éditeur(s)

Société de philosophie du Québec

ISSN

0316-2923 (imprimé)

1492-1391 (numérique)

Découvrir la revue

Citer ce compte rendu

Bonin, P.-Y. (1998). Compte rendu de [Ronald Dworkin, « Assisted Suicide : What the Court Really Said ", The New York Review of Books, 25 septembre 1997, p. 40-44.] Philosophiques, 25(2), 306-311. https://doi.org/10.7202/027499ar d'utilisation que vous pouvez consulter en ligne.

https://apropos.erudit.org/fr/usagers/politique-dutilisation/ 
Ronald Dworkin, "Assisted Suicide: What the Court Really Said ", The Vew York Review of Books, 25 septembre 1997, p. 亿0-44.

Ceux qui sont préoccupés par l'euthanasie et ceux qui s'intéressent à la philosophie morale ont sans doute pris connaissance de l'intervention des six philosophes américains bien connus en. faveur du suicide assisté. Il s'agit d'un mémoire amicus curiae présenté à la Cour suprêne des États-Unis, dont les signataires, qui tiennent à souligner qu'ils ont tous " activement contribué à son ćlabolation ", sont Ronald Dworkin, Thomas Nagel, Robert Nozick, Jolm Rawls, Thomas Scanlon et Juclith Jarvis Thompson. Le mémoire est reproduit intégralenent dans le New Fork Review of Books du 27 mars 1997 (p. 13-17), et précédé d'une introduction-résumé de Ronald Dworkin (p. 41-亿2.

Je salue l'intervention des philosoplees et je tiens d'embléc à signaler que j'approuve leur conclusion. Des rlispositions juridic|ues devivent permettre aux malarles chronicpues qui le désirent de mettre lin à leur agonie. Je considère cependant dange-

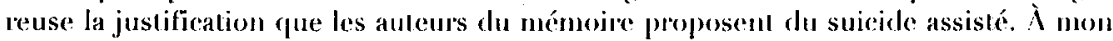
avis, une justification reposant sur l'autonomic ou les droits humains fondanentaux permet d'étendre la pratique du suicide assisté à de trop nombreuses catégories de citoyens.

Après avoir situé l'intervention des philosophes dans son contexte, j'exposerai l'essentiel de leur argumentation et discuterai sa portée. Pour d'autres critiques de la position des six philosophes, voir Michael Sandel, "Last Rights ", The New Republic, 14 avril 1997, p. 27, et Michael Walzer, "Feed the Face ", The Vew Republic, 9 juin 1997 , p. 29.

\section{Le contexte}

Les philosophes ont choisi d'intervenir au moment où les juges de la Cour suprême des États-Unis s'apprêtaient à rendre un jugement susceptible de décider pour plusieurs années de la constitutionnalité des lois des États interdisant le suicide 
assisté des malades en phase terminale. Les deux causes sur lesquelles devait porter le jugement opposaient les États de New York et de Washington à deux groupes de patients en phase terminale et à leurs médecins. Ces demiers, qui contestaient la constitutionnalité des lois des États interdisant la pratique du suicide assisté, avaient déjà obtenu des jugements favorables de la part des tribunaux des États impliqués Ronald Dworkin, "Sex and Death in the Courts ", New York Review of Books, 8 août 1996). Portées en appel devant la plus haute cour du pays, ces causes devenaient l'objet l'um enjeu considérable. Si la Cour suprême donnait raison aux malades et à leurs médecins, la praticue du suicide assisté serait dorénavant considérée constitutionnelle el cinquante États américains devraient revoir leur législation. Les libéraux craignaient cependant que la Cour suprême actuelle, plutôt conservatrice, renverse les décisions libérales des cours inferricures el laisse tomber ceux qui ont besoin de ce droit pour abreger leurs souffrances $(\mathrm{p} .41$.

Saisissant l'oceasion d'orienter la légistation américaine dans la voic qui leur paraissait la plus raisonnable, six philosophes ont soumis à la Cour suprêne un mémoire exposant leur position sur la constitutionnalité du suicide assisté ainsi que sur sa moralité. Le mémoire des philosophes répondail done à rleux ruestions : une question jurjolicpee, "Est-ee que la Constitution amérieane recomnaît le droit au suicicle assisté?"; une question morale, "Est-il momalement acceptable de permettre le suicicle assistó ?"

En juin 1997, la Cour suprême américaine a tranché la question juridique en donnant raison aux États Supreme Court Reporter, vol. 117, ${ }^{\circ} 18,15$ juillet 1997 , p. 2258-2312, Par un vote de 9-0, les juges ont estimé que le droit au suicicle assisté ne constituait pas un droit fondamental protégé par la Constitution américaine et que les États possédaient des raisons légitimes et valables d'interdire cette pratique. En ce qui concerne la question morale, comme on pourait s'y attendre, le jugement de la Cour suprême laisse le clébat owvert. Il ne relève pas de son autorité de se prononcer sur la moralité de pratiçues aussi controversées. Dans les pages qui suivent, je discuterai ce second aspect du mémoire des philosophes at soumettrai leur argumentation à la milicpue.

\section{L'argument}

L'argument de fond des philosophes an laveur du suicicle assisté, répéte à

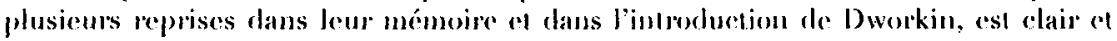
simple:

P1) Dans une société libre, les individus doivent pouvoir prendre eux-mêmes, à partir de leur foi, de leur conseience de le leurs convictions, les récisions personnelles les plus inmortantes de leur vie ip. 43 el 47 ;

P'2) Le choix du moment et de la manjère de mourir est une des décisions personnelles les plus importantes de la vie ip. 43 el 47 ;

C) Les malades en phase terminale doivent pouvoir exereer un contrôle sur le: monent el ta maniere de momir (p. 43 el 47 ).

La première prémisse énone le dogme central de la pensée momale el politique libérale. Parmi les clécisions personnelles les plus impontantes, la tradition libérale inclur généralement le choix de sa religion, de sa morale, de se marier ou non, d'avoil ou non des enfants, etc. p. 43). (Les philosophes rappellent que la Cour suprême américaine a déjà reconnu que le clroit à la liberté explicitement accordé par la Constitution comprenait le droit de faire des choix personnels et intimes. Voir Planned Parenthood os Casey, 505 U. S. 833, 851, 1992.) Les philosophes souhaitent ajouter à cette liste le droit d'exercer un contrôle sur le moment et la manière de mourir. 
La formulation la plus expressive de la seconde prémisse se trouve à la page 44 . Elle mérite d'être citée au complet.

L Lintérêt qu'uue personnc porte, au terme de sa vie. à faire des clıoix qui rellètent ses propres convictions constitue une partie si centrale de son droil plus général de faire des a choix persomels et intimes * "frime protection insuflisante de cet intéret particulier minerait son droit général. Pour chacun de nous, la mort ligure parmi les événenconts les plus importints

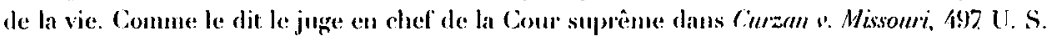
261. 281 (1990). " le choix entre la vie et la mort est une décision profondénent persounelle d'évidente et d'écasante finalité a la plupart d'entre nous considerent la mort. quel que soit. ce que nous pensons qui lui sucrédera, comme lacte final du drane de la vie, et nous voulons que ce demier acte reflete nos propres compictions, celles que nous avons essayc de suive

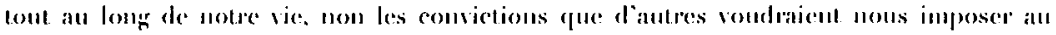
moment oì nous sommes fe pilus valuérables. inaduction libme

Cas mots som très forts el possident des implicalions que les philosophes devent assumer le moment venu.

La conclusion est égalentent énoncée ave fore et clarté Le but du mémoire est de demander à la Cour suprême de reconnaître le droit au suicide assisté à des malades chroniques agonisant en d'insupportables souffrances. $\lambda$ la fin de leur mémoire, pour faire prendre conscience de l'importance et de l'urgence de la situation, les philosophes décrivent les cas de malades dont l'intolérable condition nous est malheureusement devenue trop familière.

L'argument est de toute évidence valide. Ce qui est inquiétant est qu'il semble justifier non seulenent le droit au suicide assisté pour les malades en phase terminale, mais aussi pour de nombreuses autres catégories de citovens, en lait pour tous ceux qui, après y aroir mûrement rélléchi, décidlent de mettre fï à leur vie. Comple tenu de la force avee laquelle sont formulées et défendues les deux prémisses, je ne wis pas comment on pourrait résister à un tel élargissement de la conclusion de l'argumentation des "six". Si le droit de clioisir sa nort est une des décisions persomelles les plus importantes re la vie, pourquoi ne pas acoorler ce droit à tous les arlultes?

Les six philosophes sont conscients du danger de dérapage et une bonne partie de leur mémoire est consacrée à calmer les incuiétudes que suscite la recomnaissance d'un droit au suicide assisté. Dans son introduction, Dworkin fait d'ailleurs ressortir deux types de glissement néfastes identifiés par les auteurs ru mémoire (p. 41 ) : le glissement pratique et le glissement hérique. Le premier glisse des malades consentants aux malarles dont le consentement est forcé ou mal avisć. Le seconrl glisse des malades en phase lerminale à d’autres calégories de citopens, par excmple l'arlolescent en peine d'amour ou le handicapé. L.'examen de l'allitude des philosophes face au risque de golissement du premier ype montrem que leur défense du droil all suicide assisté est vulnérable quant au risque de glissement du second type.

\section{Le dissement pratique}

Le premier danger a été formulé par le solliciteur général lors des audiences publiques tenues par la Cour suprême. Bien que le solliciteur général reconnaisse que l'on puisse interpréter la Constitution américaine comme incluant implicitement le droit au suicide assisté, les États ont selon lui des raisons cle croire que l'exercice de ce droit, même encadré par la réglementation la plus sévère, mettrait en danger la vie d'un certain nombre de patients. Soit qu'ils pourraient se tromper sur leur propre intérêt, soit être indûment influencés par des proches (p. 45).

Les craintes du solliciteur général n'inquiètent pas les six philosophes pour les trois raisons suivantes : 1) les risques d'erreur sont également présents dans les cas de 
refus de traitement et de débranchement d'appareils assurant la survie des malades, c'est-à-dire les cas d'euthanasie passive. Or, la Cour suprême américaine a dans le passé, dans la cause Cruzan us Missouri, reconnu le droit à l'euthanasie passive. Les philosophes ne croient pas que la distinction entre commettre un acte et s'abstenir d'en commettre un, e'est-à-dire entre euthanasie passive et active, soit pertinente. (Pour la discussion de celte délicate question, je renvoie le lecteur aux pages 42 et 45 du mémoire des a six ", à la eritique de George P. Fletcher ainsi qu'à la réponse de Dworkin aux pages 45 et 46 du numéro du 29 mai 1997 du New York Review of Books.j 2) Aueune recherehe ne confirme qu'il est impossible de mettre au point une róglementation limitant les risques. $\Lambda$ u contraire, l'expérience aequise lors de l'application du jugement Cruzan a montré qu'il est possible de réglementer cette pratique. 3) Les risques évoqués par le solliciteur général ne sont pas de nature à empêcher l'exercice d'un droit. Il y a deux sortes de risques : les risques d'erreur médicale, incluant les erreurs dans l’appréciation de la maladie et de la compétence du malade, et les riscues d'infhuence indue ou perverse de la famille ou des proches. Pour les philosophes, ces deux sorles de risques sont rles risques inhérents à l'exercice de tous les droits el ne peuvent servir à justilier une interdiction absolue de lexercice d'un droit fondamental.

Les philosophes profitent de cette discussion pour signaler qu'en ce qui concerne l'évaluation des risques d'erreur el d'abus, le fardeau de la preuve incombe à ceux qui veulent linniter la pratique du suicide assisté. Étant donné que cette pratique se trouve prolégée par la Constitution, il faudiait des statistiques non équivorjues pour justifier sa limilation. Les philosophes comparent ce fardeau à celui qui incombe à ceux qui désirent limiler la liberté d'expression ou le droit à un procès équitable ip. 46.).

La réponse des philosophes au danger de glissement pratique me semble pertinente et convaincante. Elle contribue cependant à augmenter les risques de glissement théoricque.

\section{Le glissement theorique}

Je rappelle que te danger de glissement théorique repose sur le risque que la pratique du suicirbe assisté soit étendue à d'autres catégories cle citoyens que les malades en phase teminale, par exemple aux déprimés et aux bandicapés. Les "six" ne s'étendent pas beaucoup sur ce deuxiène type de démage. Ils se contentent de deux breves et sumprenantes remarques. Ils comviennent d'abord qu'il peut otre maisombable de limiler la pratique du suicide assisto alun de probeger les individus contre eux-mêmes. L'intervention du gouremement pourail se justilier daus les cas où l'́́tat possìde toutes les raisons de croire que les ciloyens concenés seraient plus lard reeonnaissants qu'on les ait empechés de s'enlever la vie p. 17. Linsuite, les " six " ćvitent la question de fond en signalant que la Cour suprêne, daus le jugement qu'elle s'apprêle à renclee au sujet des deux cas qui lui onl élé soumis, n’a pas à disculer juscfu'où l'élat pourrait intervenir pour limiter la praticpue du suicide assisté. Elle ne doit disposer que des eas qui lui ont été soumis. Dans son introduction, Dworkin résume la position des " six " et ajoute (p. 11) que les mombreux el délicats problemes posés par l'extension de la praticjue du suicide assisté ne devraicut pas empêcher la Cour suprême d'aller de l'avant.

L'attitude des philosophes face au risque de glissement théorique laisse perplexe et n'est pas de nature à calmer les inquićturles. Elle illustre clairement la situation dólicate où l'argumesntation "autonomiste s conduit ses partisans et met en lumière les principales laiblesses et contradictions dlu mómoire des a six ".

Premièrement, la simple reconnaissance d'un danger de glissement théorique pose un problème aux " autonomistes ". Si l'autonomie constitue réellement la base de la défense du suicide assisté, la préoccupation principale de l'État ou du législateur 
derrait alors êre de s'assurer que la décision des citoyens concernés est libre et unurement rófléche. Tous les problèmes liés au glissement théorique devaient ètre interprétés comme des difficultés soulevées par le risque de glissement pratique. Par excmple, les problemes posés par le cas de l'adolescent en peine d'amour ou celui d'une personne devenue handicapée à la suite d'un accident devraient être traités uniquement conme des problemes concernant la valeur de la reffexion précédant leur décision. La seule façon, pour les autonomistes conséquents, d'empêdner ces individus de mettre leur plan à exécution serait de montrer que leur décision, prise sous le coup d'une émotion passagère, n'est pas le fruit d'une décision mûrement réfléchie. Faire tout reposer sur le consentement éclairé des individus est rependant risgué, car il est facile d'imaginer des eas oi il ne sera pas aisé de déteminer si la décision posside le niveau de réflexion requis.

Deuxiènement, une fois que les risques de ghlissement théorique sont interprélés comme des risques de glissement pratique, tous les arguments imorgués par les " six" pour réduire ou minimiser les riscues de glissement paticgue deviement alors autan l'arguments pour justifier l'extension du suicide assisté et augmenter les riscues de démage : 1 les risques d'erreur sont présents dans loutes les formes de suicide assisté; 2 il est possible de régglementer la pratique ion pourrail, par exemple, limiter l'exercice de ce droil aux citovens ayant suivi une psvchothérapie ou à ceux âgés de plus de trente ans: ; 3 s'agissant d'un droit fondamental, les risques d'erreur ne somt pas suffisants pour empêcher l'extension de la pratique du suicide assisté.

Troisièmement, la règle paternaliste que les six philosophes sont prêts à considérer pour limiter la pratique du suicide assisté est cléconccrtante puisque peu compatible avec leur vigoureuse défense "autononiste " chu suicide assisté. L'adoption d'une règle générale permettant à l'État de limiter les droits ot libertés de base des citoyens lorsque les représentants de l'État possèdent de bonnes raisons de croire que les citovens leur en seraient reconnaissants ferait une moquerie des idées de droit fondamental et d'autonomic. Si l'autonomie signifie considérer l'individu comme l'arbitre suprême de ce qui est bon pour lui, autoriser l'intervention de l'́tat chacfue fois que la majorité de la population consiclère qu'il est préférable pour l'individu ial faut préciser que te bien de la société n'est pas en cause icil de s'abstenir de certains comportements anéantit à toutes fins utiles l'autonomic des indivichus. Les implications de cette règle, entre autres pour la consommation daalcool, de labac et de matériel pornographique, pourraient être considérables.

Quatrièmement, s'il est vrai que la Cour suprême des Élats-Unis u'a pas pour le moment à se prononcer sur la délimitation du droit au suicide assisté, il n'en est pas moins vrai que dans le cas où la Cour reconnaîtrait ce droit, la justification retenue aurait de lourdes conséquences sur la pratique du suicide assisté. Si la Cour suprême fait sienne la justification proposée par les six philosophes, elle deva éventuellement trancher des cas extrêmement délicats ne mettant pas en cause cles malarles en phase terminale. La Cour devra décider du sort des personnes atleintes de la maladie d'Alzheimer, des paraplégiques, de ceux, de plus en plus nombreux, qui ne peuvent psychologiquement pas supporter d'être atteints d'une maladie incurable, de l'artiste qui considère comme finie sa vie créatrice et de tous ceux qui, pour une raison ou une autre, en ont assez de vivre. Pourquoi ces personnes n'auraient-elles pas le droit de prendre elles-mêmes une des décisions persommelles les plus importantes de leur vie ?'

En résumé, les six philosophes font face au dilemme suivant : s'ils acceptent de limiter considérablement la pratique du suicide assisté, ils montrent qu'ils ne prennent pas au sérieux le droit de choisir le moment et la manière de mourir sur lequel repose leur argumentation ; s'ils refusent, leur proposition devient très inquiétante. 


\section{Une meilleure approche}

Je terminerai en précisant que la principale difficulté soulevée par la position des " six • n'est pas que la reconnaissance d'un droit au suicide assisté pour les malades chroniques ou en phase terminale risque d'étendre une pratique controversée. Toutes les politiques sociales et tous les droits présentent des risques d'élargissement indu. La difficulté est plus exactement que l'argument dont les philosophes se servent pour justifier le suicide assisté des malades chronicues multiplie les risques de dérapage. S'il n'y avait que la justification "aulonomiste ", ceux qui appuient le droit au suicide assisté des malarles chronicpues rlevraient peutềtre se résigner et en assumer les conséquences. Ce llest cependant pas le cas. Il existe d'autres défenses de ce droit. On pourrait, par exemple, fourler le droil au suicide assisté directement sur la volonté de mente un tcrme à la souffrance des agonisants et de préserver la dignité des malades.

Je n'ai pas l'intention de développer ici cettc voie, l'objectif du présent essai étant essentiellement de commenter la position des six philosophes américains. Je signalerai cependant deux des principaux avantages de cette conception sur la justification " autonomiste " privilégiée par les a six ". Premièrement, en ciblant des groupes précis de citoyens, en l'occurrence les malades chroniques ou en phase terminale, une justification basée sur la volonté de mettre fin à ume souffrance inutile et de préserver la dignité cles malades permet de limiter considérablement les risques de glissement théorique. Elle permet d'ćviter les problèmes liés à la gestion de principes généraux difficiles à formuler et à appliquer.

Deuxièmement, n'en déplaise aux six philosophes, dans les sociétés libérales et clémocratiques occirlentales il semble exister un plus large consensus sur la nécessité d'éviter Ja douleur extrême et d'assurer la dignité des grands malades que sur le droit de choisir le moment et la manière de mourir. Pour ceux qui considèrent yu'il est clans l'intérêt de tous les citoyens d'une démocratie pluraliste de rechercher les plus larges consensus possibles et de limiter les occasions de désaccord, cet avantage n'est pas négligeable.

Pierre-Yves Bonin

Département de philosoplie

Université du Québec à Trois-Rivières 